\title{
Practice-changing publications: Muscle-invasive bladder cancer
}

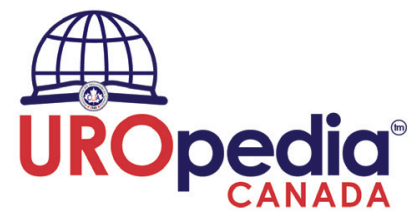

\author{
Khatereh Aminoltejari, MD'; Girish Kulkarni, MD'; Ross Mason, $M D^{2}$ \\ 'Division of Urology, University of Toronto, Toronto, ON, Canada; ${ }^{2}$ Department of Urology, Dalhousie University, Halifax, NS, Canada
}

Cite as: Aminoltejari K, Kulkarni G, Mason R, et al. Practice-changing publications: muscle-invasive bladder cancer. Can Urol Assoc J 2022;16(1):E57-60. http://dx.doi.org/10.5489/cuaj.7733

The CUA has introduced a webinar series called PracticeChanging Publications (PCP series), which features

Canadian experts discussing recent data that have impacted their field. The CUAJ is proud to present summaries of these talks and the important research discussed in this series.

If you missed any of the webinars, you can access them at https://www.cua.org/UROpedia and earn all-important CPD Section 3 credits!

\section{PURE-01 trial: Neoadjuvant immunotherapy in treatment of muscle-invasive bladder cancer}

Radical cystectomy (RC) and bilateral pelvic lymph node dissection (bPLND) are a standard of care treatment for localized muscle-invasive urothelial bladder carcinoma (MIBC). Neoadjuvant chemotherapy has been shown to improve overall survival in this setting, but it is underused due to patient and provider factors and preferences. ${ }^{1,2}$ Immune checkpoint inhibitors have revolutionized the metastatic and locally advanced disease space in urothelial carcinoma and, in 2018, the PURE-01 trial published its investigation on the role of pembrolizumab as neoadjuvant immunotherapy before RC. ${ }^{3}$ This open-label, single-arm, phase 2 study by Necchi and colleagues enrolled 50 patients across two centers in Milan, Italy. All patients had CT2-4aNOMO MIBC and were scheduled for RC. The primary endpoint of the study was the pT0 rate in the intention-to-treat population. ${ }^{3}$ Patients received three courses of pembrolizumab $200 \mathrm{mg}$ intravenously every three weeks followed by RC 1-3 weeks after the last dose. The authors found that pembrolizumab resulted in $42 \%$ of patients $(21 / 50)$ having pT0 at the time of their RC and a further six patients having pTa-T1, for a total of $54 \%$ of patients being down-staged to non-MIBC. ${ }^{3}$

A 2020 update to the original study included patients with predominantly variant histology $(\mathrm{VH})$ in the protocol. ${ }^{4}$ Defined as $>50 \% \mathrm{VH}$ of urothelial carcinoma $(\cup \mathrm{C})$ in the tumor, these predominantly $\mathrm{VH}$ tumors typically have a more aggressive clinical course. Pathological complete response (pT0) was the primary endpoint. ${ }^{4}$ The study enrolled 114 patients with $30 \%(n=34)$ having $\mathrm{VH}(17 \%$ with predominant $\mathrm{VH})$. The most common variant was squamous cell carcinoma (SCC). In total, the pT0 rate was 37\% (95\% confidence interval $[\mathrm{Cl}] 28-46)$ and the $\mathrm{pT} \leq 1$ rate was $55 \%(95 \% \mathrm{Cl}$ 46-65). It was notable that patients with predominant VH had much lower overall pT0 rates at $\mathbf{1 6} \%$. However, among those with SCC $(n=7), 6 / 7$ patients were downstaged to $\mathrm{pT} \leq 1$, with one achieving pT0 stage. Additionally, of those with lymphoepithelioma-like variants, 2/3 achieved pT0 stage

Concurrently, results from PURE-01 on the surgical safety of performing RC and bPLND after neoadjuvant treatment with pembrolizumab were published. A total of 68 patients who had neoadjuvant therapy underwent RC $-77 \%(n=52)$ robot-assisted and $23 \%(n=16)$ open procedures - of whom, a total of $31(46 \%)$ received an orthotopic neobladder. ${ }^{5}$ Patients were interviewed at 90 days post-RC to report complications. A total of $52(77 \%)$ patients experienced anygrade complications, $47(69 \%)$ had grade $\geq 2$ complications, and $22(32 \%)$ were re-admitted within 90 days. ClavianDindo grade $\geq 3$ (high-grade) complications occurred in 23 (34\%) patients and no mortalities were reported at 90 days. The authors noted that these results are similar to those expected in all-comers undergoing RC. Overall, this study provided the first prospective evidence in support of the surgical safety of RC and bPLND after neoadjuvant immune checkpoint inhibitor therapy in patients with muscle invasive, non-metastatic bladder cancer. ${ }^{5}$

\section{RAZOR trial: The debate between robotic vs. open cystectomy}

Open RC and urinary diversion are complex surgical procedures with significant risks of intra- and perioperative morbidity and mortality. ${ }^{6}$ Robot-assisted radical cystectomy (RARC) has been touted as an alternative approach. The RAZOR trial, published in 2018, is the largest prospective, randomized study comparing the two surgical techniques. ${ }^{7}$

In an open-label, non-inferiority, phase 3 trial performed in 15 medical centers in the U.S., Parekh and colleagues randomized (1:1) patients with clinical stage Tis-T4, N0-N1, 
MO bladder cancer to open RC or RARC. ${ }^{7}$ The primary endpoint was two-year progression-free survival (PFS), with noninferiority established if the lower bound of the one-sided $97.5 \% \mathrm{Cl}$ for the treatment difference (robotic cystectomy minus open cystectomy) was greater than -15 percentage points. A total of 302 patients were included in the final analysis, with 150 in the robotic cystectomy group and 152 in the open RC group. ${ }^{7}$ PFS at two years was $72.3 \%(95 \%$ $\mathrm{Cl} 64.3-78.8)$ in the RARC group and $71.6 \%(95 \% \mathrm{Cl} 63.6-$ 78.2 ) in the open group (difference $0.7 \%, 95 \% \mathrm{Cl}-9.6 \%$ to $\left.10.9 \% ; p_{\text {non-inferiority }}=0.001\right)$, which indicated non-inferiority of robotic cystectomy. Complication rates between the two groups were also similar at $67 \%$ in the RARC arm and $69 \%$ in the open RC arm.

An update to the RAZOR trial reported on the healthrelated quality of life (HRQoL) in patients in the RARC and open RC group. ${ }^{8}$ They used the Functional Assessment of Cancer Therapy (FACT)-Vanderbilt Cystectomy Index (VCI) and the Short-Form 8 Health Survey (SF-8) at baseline, as well as at three and six months postoperatively. ${ }^{8}$ The primary objective was to assess the impact of surgical approach on HRQoL and secondarily assess the impact of the type of urinary diversion on HRQoL. ${ }^{8}$

No statistically significant difference was noted at any time point for all FACT-VCl outcomes. ${ }^{8}$ In composite scores, both open RC and RARC showed significantly improved emotional well-being scores at three and six months postoperatively. ${ }^{8}$ Similarly, no differences were noted in the SF-8 scores between robotic and open cystectomy arms. ${ }^{8}$ In the exploratory analysis of urinary diversion type, there was no statistically significant difference between continent and non-continent diversions, except for the Functional Assessment of Cancer Therapy-Bladder-Cystectomy questionnaire score (FACT-BL-Cys), a component of the FACT$\mathrm{VCl}$, which was lower for the continent diversion type by 5.5 points at three months $(p<0.01) .{ }^{8}$ In assessment of the SF-8 components, authors found the only difference between the two groups was with the physical component summary (PCS) score, as patients with continent urinary diversion showed improvement at six months by 4.6 points compared to noncontinent diversion $(p=0.043) .{ }^{8}$

Overall, the RAZOR trial established the non-inferiority of robotic and open surgical approaches to $\mathrm{RC}$ with respect to oncological outcomes. No differences were seen in rates or severity of postoperative complications and the patients' HRQoL was not impacted by the choice of operative technique. ${ }^{8}$

\section{The role of multiparametric magnetic resonance imaging in staging localized bladder cancer}

The Vesical Imaging-Reporting and Data System (VI-RADS) is a five-point scale aimed at improving preoperative staging of bladder cancer (BCa). ${ }^{9}$ In 2020, Del Giudice and colleagues published a large, single-center, prospective cohort study to validate the diagnostic accuracy of VI-RADS and assess its clinical impact on management of newly diagnosed BCa. ${ }^{10}$ Patients with suspicion of BCa were offered a multiparametric magnetic resonance imaging (mpMRI) prior to transurethral resection of bladder tumor (TURBT). The primary endpoint was assessment of sensitivity, specificity, positive predictive value (PPV), and negative predictive value (NPV) of the VI-RADS score in predicting NMIBC and MIBC at initial TURBT. The secondary endpoint was to assess the aforementioned parameters in patients who underwent a re-TURBT due to the presence of high-risk Ta-T1 NMIBC. ${ }^{10}$

A total of 231 patients were enrolled to undergo a mpMRI prior to TURBT. Of these, 114 patients required re-TURBT due to finding of high-risk NMIBC and 42 were found to have MIBC after the first TURBT. A threshold of VI-RADS $\leq 2$ was used as a clinical predictor of non-(N)MIBC at reTURBT, while VI-RADS score of $\geq 3$ was used as predictor of under-staged MIBC at TURBT. ${ }^{10}$ The sensitivity and specificity of $\mathrm{mpMRI}$ in discriminating between NMIBC and MIBC was 91.9\% (95\% Cl 82.2-97.3) and 91.1\% (95\% Cl 85.8-94.9), respectively. ${ }^{10}$ The PPV and NPV were $77.5 \%(95 \% \mathrm{CI} 65.8-$ 86.7) and $97.1 \%(95 \% \mathrm{Cl} 93.3-99.1)$, respectively. ${ }^{10}$

Among the 114 patients that required re-TURBT, 28 $(24.5 \%)$ had no detrusor muscle in the first TURBT specimen. Of these 28 , eight $(28.6 \%)$ were high-grade (HG) Ta, $11(39.3 \%)$ were HG-T1, and nine $(32.1 \%)$ were low-grade (LG)-T1. At re-TURBT, 58 (50.9\%) patients had no cancer, $36(31.6 \%)$ were diagnosed with persistent HR-NMIBC, and $20(17.5 \%)$ were upstaged to MIBC..$^{10}$ Multiparametric MRI before re-TURBT demonstrated sensitivity and specificity of 85\% (95\% Cl 62.1-96.8) and 93.6\% (95\% Cl 86.6-97.6), respectively, to identify patients diagnosed with MIBC. The authors concluded that mpMRI using the VI-RADS system appears to have high accuracy for predicting MIBC at both initial and repeat TURBT and can be used to identify falsenegative cases that may obviate the need for repeat TURBT. ${ }^{10}$ On the other hand, the significant portion of patients found to have residual disease at re-TURBT still bolsters the case for repeat TURBT as standard of care in HR-NMIBC. ${ }^{10}$

\section{The POUT trial: Adjuvant chemotherapy in upper tract urothelial carcinoma}

Upper tract urothelial carcinoma (UTUC) is a rare disease and more often presents as muscle-invasive disease as compared to urothelial carcinoma of the bladder. In 2020, Birtle and colleagues published the results of a phase 3, open-label, randomized, controlled trial that was designed to assess the efficacy of adjuvant systemic platinum-based chemotherapy in patients with UTUC. ${ }^{11}$ They recruited patients with UTUC 
after nephroureterectomy staged as either pT2-4, pN0-N3 M0 or pTany, N1-3 and M0 across 71 hospitals in the U.K.

The study randomized 261 patients with a 1:1 allocation to either chemotherapy $(n=132)$ or surveillance $(n=129)$. The chemotherapy regimen involved four 21-day cycles of cisplatin or carboplatin (for glomerular filtration rate $<50 \mathrm{~mL} / \mathrm{min}$ but above $\geq 30 \mathrm{~mL} / \mathrm{min}$ ), initiated within 90 days of surgery. The primary endpoint was disease-free survival. Secondary endpoints included metastasis-free survival, overall survival, treatment compliance, acute toxicity, late toxicity, and patient-reported quality of life. ${ }^{11}$ The patients were relatively evenly matched across chemotherapy and surveillance, with a notable exception in pathological stage: a pT2 distribution of $23 \%$ vs. $34 \%$ and a pT3/4 rate of $77 \%$ vs. $67 \%$ randomized to surveillance vs. chemotherapy, respectively.

The authors found that the three-year disease-free survival was significantly improved $(71 \%$ vs. $46 \%)$ with chemotherapy, with an estimated absolute difference of 25\% (95\% Cl $11-38) ; 44 \%$ of the chemotherapy arm had grade 3 or worse adverse events. Recruitment in this trial stopped early, as an interim analysis demonstrated that treatment with chemotherapy initiated within 90 days of nephroureterectomy was beneficial enough to meet a priori defined early stopping criterion for efficacy. Patients in the observation arm who were within the 90-day window were recommended to undergo chemotherapy given the clear disease-free survival benefits. ${ }^{11}$ This trial provides strong support in favor of adjuvant chemotherapy as standard of care in patients with locally advanced urothelial UTUC.

\section{Health-related quality of life for patients treated with trimodal therapy}

The BC2001 trial, the largest randomized trial of radiotherapy in MIBC, had previously reported that the addition of 5-FU and mitomycin C chemotherapy to radiation therapy significantly improved survival outcomes. ${ }^{12}$ In 2020, the patient-reported, HRQoL outcomes of this trial were published. In this phase 3 trial, after screening, 458 patients were randomized across 45 U.K. hospitals $1: 1$ to radiation with chemotherapy (CRT) or radiotherapy (RT) alone and additionally randomized to standard whole-bladder radiotherapy (stRT) or reduced high-dose volume radiotherapy (RHDVRT) with tumor boost. ${ }^{13}$ The primary outcome for both cRT and RT was the HRQoL change from baseline in the bladder cancer subscale (BLCS) of the FACT-BL questionnaire at 12 months. The FACT-BL incorporates five categories: physical, social, emotional (EWB), and functional well-being, as well as the BLCS. Secondary outcomes included changes from baseline in total FACT-BL score, Trial Outcome Index (TOI), defined as a sum of physical and functional well-being scores, as well as separate component subscales of the FACT-
BL scores. Patients completed the questionnaire at baseline, at the end of treatment, at six months from end of treatment, and then annually to five years. ${ }^{13}$ They also explored the effects of pre-treatment with neoadjuvant chemotherapy in a non-randomized comparison between pretreated and nonpretreated patients.

For the whole BC2001 population, immediately after treatment, there was a significant change in BLCS score of -5.06 (from baseline 0), a mean change in total score of -8.22 (where the baseline is set at 0 ). By six months after $\mathrm{RT}$, all subscales had improved or returned to baseline, and by one year, all subscales had either remained at baseline or improved. Overall, there was no significant difference in the BLCS score at one year when comparing CRT and RT (adjusted difference 0.18,95\% Cl -1.60-1.96; $\mathrm{p}=0.8$ ). In addition, the other subscales did not show significant differences at any time point. Similarly, the difference between stRT and RHDVRT at one year from baseline was $2.0(95 \%$ $\mathrm{Cl}-0.31-4.33$; $\mathrm{p}=0.089$ ), which indicated no significant difference between the two groups; however, the trend in BLCS at five years was a worsening BLCS in the RHDVRT group as compared to stRT group (not statistically significant). Interestingly, for the overall trial population, the EWB score significantly improved from baseline at the end of treatment, with a mean change of $1.14(99 \% \mathrm{Cl} 0.68-1.16$; $\mathrm{p}<0.001)$ and remained above baseline in followup.

Overall, in this largest study of HRQoL in patients who were undergoing bladder preservation treatment for MIBC, authors showed that patients had an initial reported detriment to their quality of life but returned to baseline within six months of treatment with trimodal therapy. ${ }^{13} \mathrm{Also}$, the addition of concomitant chemotherapy or neoadjuvant chemotherapy did not significantly impact $\mathrm{HRQOL}$, which supports the routine use of $5-\mathrm{FU}$ and mitomycin $\mathrm{C}$ in trimodal therapy. ${ }^{13}$ Finally, the lack of a difference in HRQoL for stRT compared to RHDVRT suggests that whole bladder RT does not contribute to worse bladder function, obviating the need for reduced-dose RT schemes.

Competing interests: Dr. Kulkarni has been an advisory board member for Astellas, Ferring, Janssen, Merck, Roche, and Theralase; has received grants/honoraria from Abbvie, Ferring, Sanofi, and TerSera; and has participated in clinical trials supported by Astra Zeneca, Bristol Myers Squibb, Janssen, Merck, and Theralase. Dr. Mason has been an advisory board member for Abbvie, Sanofi, TerSera, and Verity; a speakers' bureau member for Abbvie, Astellas, and TerSera; received a grant from CUOG/Bladder Canada Canada; and has participated in several clinical trials relating to the management of kidney, bladder, and prostate cancer. Dr. Aminoltejari does not report any competing personal or financial interests related to this work. 
Aminoltejari et al

\section{References}

1. Sonpavde G, Goldman BH, Speights VO, et al. Quality of pathologic response and surgery correlate with survival for patients with completely resected bladder cancer after neoadjuvant chemotherapy. Cancer 2009;115:4104-9. hitps://doi.org/10.1002/cncr.24466

2. Bellmunt J, de Wit R, Vaughn DJ, et al. Pembrolizumab as second-line therapy for advanced urothelial carcinoma. N Engl J Med 2017;376:1015-26. https://doi.org/10.1056/NEJMoal613683

3. Necchi A, Anichini A, Raggi D, et al. Pembrolizumab as neoadjuvant therapy before radical cystectomy in patients with muscle-invasive urothelial bladder carcinoma (PURE-01): An open-label, single-arm, phase 2 study. J Clin Oncol 2018;36:3353-60. htrps://doi.org/10.1200/JC0.18.01148

4. Necchi A, Raggi D, Gallina A, et al. Updated results of PURE-01 with preliminary activity of neoadjuvant pembrolizumab in patients with muscle-invasive bladder carcinoma with variant histologies. Eur Urol 2020;77:439-46. https://doi.org/10.1016/i.eururo.2019.10.026

5. Briganti A, Gandaglia G, Scuderi S, et al. Surgical safety of radical cystectomy and pelvic lymph node dissection following neoadjuvant pembrolizumab in patients with bladder cancer: Prospective assessment of perioperative outcomes from the PURE-01 trial. Eur Urol 2020;77:576-80. https://doi.org/10.1016/i. eururo.2019.12.019

6. Shabsigh A, Korets R, Vora KC, et al. Defining early morbidity of radical cystectomy for patients with bladder cancer using a standardized reporting methodology. Eur Urol 2009;55:164-74. https://doi.org/10.1016/i.eururo.2008.07.031

7. Parekh DJ, Reis IM, Castle EP, et al. Robot-assisted radical cystectomy versus open radical cystectomy in patients with bladder cancer (RAZOR): An open-label, randomised, phase 3, non-inferiority trial. Lancet 2018;391:2525-36. htrps://doi.org/10.1016/S0140-6736(18)30996-6
8. Becerra MF, Venkatramani V, Reis IM, et al. Health-related quality of life of patients with bladder cancer in the RAZOR trial: A multi-institutional, randomized trial comparing robot vs. open radical cystectomy. J Urol 2020;204:450-9. https://doi.org/10.1097/JU.0000000000001029

9. Panebianco V, Narumi Y, Altun E, et al. Multiparametric magnetic resonance imaging for bladder cancer: Development of VI-RADS (Vesical Imaging-Reporting And Data System). Eur Urol 2018;74:294-306. https://doi.org/10.1016/i.eururo.2018.04.029

10. Del Giudice F, Barchetti G, De Berardinis E, et al. Prospective assessment of Vesical Imaging Reporting and Data System (VI-RADS) and its clinical impact on the management of high-risk non-muscle-invasive bladder cancer patients candidate for repeated transurethral resection. Eur Urol 2020;77:101-9. https://doi.org/10.1016/i.eururo.2019.09.029

11. Birtle A, Johnson $M$, Chester J, et al. Adjuvant chemotherapy in upper tract urothelial carcinoma (the POUT trial): A phase 3, open-label, randomized controlled trial. Lancet 2020;395:1268-77. https://doi.org/10.1016/S0140-6736(20)30415-3

12. James ND, Hussain SA, Hall E, et al. Radiotherapy with or without chemotherapy in muscle-invasive bladder cancer. N Engl J Med 2012;366:1477-88. https://doi.org/10.1056/NEJMoal 106106

13. Huddart RA, Hall E, Lewis $R$, et al. Patient-reported quality of life outcomes in patients treated for muscleinvasive bladder cancer with radiotherapy \pm chemotherapy in the BC2001 phase 3 , randomized, controlled trial. Eur Urol 2020;77:260-8. https://doi.org/10.1016/i.eururo.2019.11.001

Correspondence: Dr. Girish Kulkarni, Division of Urology, University of Toronto, Toronto, ON, Canada; Girish.Kulkarni@uhn.ca 